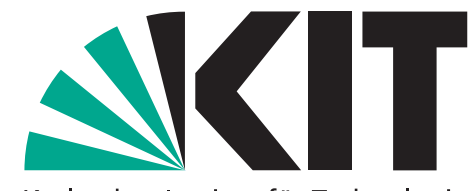

Karlsruher Institut für Technologie

\title{
A mechanism for booms and busts in housing prices
}

by Marten Hillebrand and Tomoo Kikuchi

No. 40 | MAY 2012

\section{WORKING PAPER SERIES IN ECONOMICS}

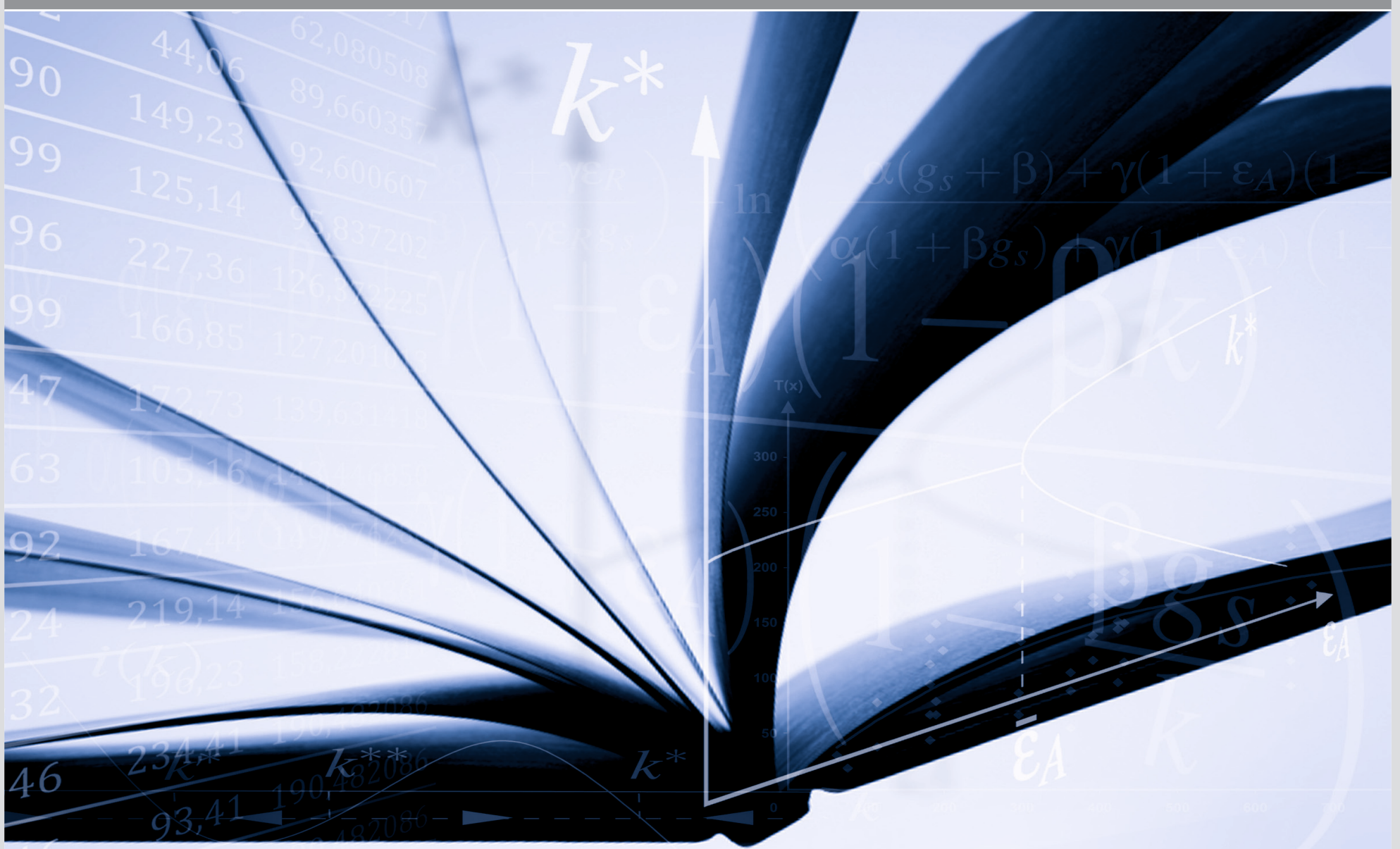




\section{Impressum}

Karlsruher Institut für Technologie (KIT)

Fakultät für Wirtschaftswissenschaften

Institut für Wirtschaftspolitik und Wirtschaftsforschung (IWW)

Institut für Wirtschaftstheorie und Statistik (ETS)

Schlossbezirk 12

76131 Karlsruhe

KIT - Universität des Landes Baden-Württemberg und nationales Forschungszentrum in der Helmholtz-Gemeinschaft

Working Paper Series in Economics

No. 40, May 2012

ISSN 2190-9806

econpapers.wiwi.kit.edu 


\title{
A Mechanism for Booms and Busts in Housing Prices*
}

\author{
Marten Hillebrand ${ }^{\dagger} \quad$ Tomoo Kikuchi ${ }^{\ddagger}$
}

April 15, 2012

\begin{abstract}
We study an exchange economy with overlapping generations of consumers who derive utility from consuming a non-durable commodity and housing. A banking sector offers loans to finance housing. We provide a complete characterization of the equilibrium dynamics which alternates between an expansive regime where housing prices increase and banks expand loans and a contractive regime associated with decreasing housing values and shrinking credit volume. Regime switches occur even under small but persistent income changes giving rise to large and recurrent booms and busts in housing prices not reflecting changes in fundamentals.
\end{abstract}

JEL classification: $\mathrm{C} 62, \mathrm{E} 32, \mathrm{G} 21$

Keywords: OLG, Housing prices, Credit volume, Boom-bust scenarios, Regime switching

*We would like to thank Costas Azariadis, Volker Böhm, John Drifill, Aditya Goenka, Basant Kapur, Hoon Hian Teck, Haiping Zhang, Shenghao Zhu, and seminar participants at NUS, Nanyang Technological University, Singapore Management University, and the AEI-Four Joint Workshop in Singapore for their helpful comments and Yan Naung Oak for computational assistance. We acknowledge financial support of the Ministry of Education, Singapore for the Academic Research Fund R-122-000-140-112.

${ }^{\dagger}$ Karlsruhe Institute of Technology, Department of Economics, Germany, Email: marten.hillebrand@kit.edu, Tel.: +49721 60845667

$\ddagger$ National University of Singapore, Department of Economics, Singapore, Email: ecstk@nus.edu.sg 


\section{Introduction}

For more than a decade, from 1997 to 2007, U.S. residential values had continually increased with housing prices in July 2007 being almost three times as high as in 1996. In mid 2007, this increase came to an abrupt halt and housing prices dropped by almost $17 \%$ until June 2008 and by more than 30\% between June 2007 and June 2009. ${ }^{1}$ In retrospect, most researchers today seem to agree that houses were overvalued in 2006/2007 (cf. Kocherlakota (2011)), i.e., the increases in housing values were not backed by fundamentals. A closer inspection of the potential reasons also reveals that the build-up of the housing bubble was accompanied by a continual increase in mortgage loans and an ever increasing mortgage debt which dropped sharply after the burst in 2007 (cf. Chen \& Winter (2011) and He, Wright, \& Zhu (2011)). This observation points to a potential linkage between the lending activities of banks and the evolution of housing prices.

While the US episode certainly motivates a number of theoretical questions, the focus of the present paper is on the co-movement between mortgage loans and housing prices. We develop a theoretical model, which explains large movements in housing prices accompanied by corresponding changes in credit volume. Our analysis uncovers a simple mechanism through which small but persistent income changes generate large movements in housing values and the aggregate credit volume. Remarkably, these movements occur in the absence of any sort of frictions. The mechanism generating these boom-bust cycles is a switch between two regimes each associated with a certain income level. In the first regime, consumers are eager to borrow and willing to pay a high interest on loans such that banks expand their credit volume over time and housing prices increase without bound. In the second regime, consumers are only willing to borrow at a lower interest for which the credit volume shrinks over time and housing prices decrease to a lower bound. A switch between these two regimes occurs under quite moderate income changes. If the change is persistent, the system tends to stay in one regime for a number of periods generating large movements in housing prices and the credit volume. During boom phases, consumers buy houses at prices they could never have afforded and take loans they would be unable to repay from their income alone. Thus, the financial side essentially decouples from the real side of the economy.

On the methodological side, our paper belongs to the literature on the pure exchange overlapping generations economy. The seminal work by Samuelson (1958) sets up the foundation of the analysis of fiat money in the pure exchange overlapping generations economy. Gale (1973) studies the dynamic formulation of the Samuelson model and our paper can best be seen as a straightforward extension of his work modified to include inside money in the form of mortgage loans, and a durable good (housing) instead of fiat money. Fiat money in Samuelson (1958) and Gale (1973) is in fixed supply and thus its relative value is bounded by the fundamentals in the economy. By contrast, the

\footnotetext{
${ }^{1}$ These figures are taken from the Casey-Shiller housing index composed over 10 areas.
} 
inside money in our model can fuel the housing price indefinitely.

In its economic theme, our paper is related to the theoretical literature on housing market dynamics. As in our paper, Ortalo-Magné \& Rady (2006) and Arce \& LópezSalido (2011) study pure exchange overlapping generations models with a durable good (housing). Ortalo-Magné \& Rady (2006) studies a four period overlapping generations model of the housing market with two types of homes, "starter" and "trade-up" homes, and a down-payment constraint on borrowing. They show that the volatility in the income of young households plays a critical role for the "excess" volatility of housing prices. Arce \& López-Salido (2011) develops a three period overlapping generations model, where bubbles solve the problem of insufficient asset supply in the presence of financial friction. They analyze how loan supply shocks can eliminate bubbles and push the economy into a low-valuation regime in which the volume of debt and the housing price are lower in the presence of multiple steady states. They also show the existence of multiple equilibria of pure and housing bubbles. Our model shares a common feature with Ortalo-Magné \& Rady (2006) and Arce \& López-Salido (2011) that housing generates utility and serves to collateralize loans. However, borrowing is limited to a fraction of the value of the housing stock in their models while our model does not assume any kind of financial frictions.

He, Wright, \& Zhu (2011) studies an infinite-horizon economy where houses, in addition to providing utility, also facilitate transactions in imperfect credit markets. They show that even when fundamentals are constant and agents are fully rational, house prices can be priced above the present value of the marginal utility from living in the house. Their housing dynamics is driven by beliefs, i.e. a self-fulfilling prophecy in the economy with multiple equilibria as well as by deterministic cycles and chaos. There are no multiple equilibria in our model nor does it exhibit deterministic cycles or chaos. In this respect, the mechanism in our model is closer to the one in Ortalo-Magné \& Rady (2006) where income shocks of the young household drives the housing price overreaction.

This paper is also related to the recent literature that focuses on the role of housing and, more generally, of durable consumption goods for the macroeconomy (e.g. Bajari, Chan, Krueger \& Miller (2010) and Chen \& Winter (2011)). Chen \& Winter (2011) evaluates the quantitative impact of the change in housing financing on the consumption boom. Bajari, Chan, Krueger \& Miller (2010) estimates a dynamic structural model of housing demand and uses it to simulate how consumer behavior responds to house price and income declines as well as tightening credit constraints.

The paper is organized as follows. Section 2 introduces the model. Section 3 derives the forward-recursive structure of equilibria while Section 4 studies the equilibrium dynamics under constant incomes. Section 5 generalizes the deterministic structure to the case with random incomes and analyzes the scope for boom-bust scenarios to emerge due to persistent income changes. We also show that a large component of housing prices is a pure bubble defined as a deviation from the fundamental value. The theoretical findings 
are illustrated and quantified in Section 6 with the help of numerical simulations. Section 7 concludes. Proofs for all results can be found in the mathematical appendix.

\section{The Model}

We consider an exchange economy with discrete time periods $t \geq 0$ and a durable and a non-durable commodity. We refer to the durable commodity as 'housing' and the non-durable good as 'the consumption good'. The latter is chosen as the numeraire.

\section{Consumption sector}

The consumption sector consists of overlapping generations of homogeneous, two-period lived consumers. Each member of the generation born in $t \geq 0$ is endowed with $e_{t}^{y}>0$ units of the consumption commodity when young and $e_{t+1}^{o}>0$ units when old. The following assumption specifies the probabilistic nature of their incomes. ${ }^{2}$

\section{Assumption 1}

The process $\left\{e_{t}\right\}_{t \geq 0}$ where $e_{t}:=\left(e_{t}^{y}, e_{t+1}^{o}\right)$ consists of random variables defined on a common probability space $(\Omega, \mathcal{F}, \mathbb{P})$ with values in $\mathcal{E}:=\left[e_{\min }^{y}, e_{\max }^{y}\right] \times\left[e_{\min }^{o}, e_{\max }^{o}\right] \subset \mathbb{R}_{++}^{2}$. The process is adapted to some filtration $\left\{\mathcal{F}_{t}\right\}_{t \geq 0}$ such that $e_{t}: \Omega \rightarrow \mathcal{E}$ is $\mathcal{F}_{t}$-measurable.

A consequence of Assumption 1 is that young consumers observe their first and second period income when they make decisions. Thus, our analysis abstracts from income uncertainty. Instead, we demonstrate below that predictable income shifts suffice to generate large movements in housing prices.

\section{Housing}

Houses are retradable and in constant supply normalized to unity. The young purchase houses at the end of period $t$ at the price $p_{t}>0$, for which they incur a fixed cost $\kappa>0$ per unit to be paid in the following period $t+1$. This parameter can be interpreted as a cost associated with holding houses such as maintenance and remodeling costs or insurance payments. Housing investment provides a possibility to transfer wealth intertemporally and yields utility in the following period.

\section{Banking sector}

The banking sector consists of a large number of banks which offer loans at a riskless interest factor $R_{t}>0$. Let $b_{t} \geq 0$ denote the aggregate credit volume corresponding to the resource available to the banking sector at time $t$. This resource is provided as loans to the young and financed by the loan repayment of the old such that

$$
b_{t}=R_{t-1} b_{t-1}, \quad t \geq 1 .
$$

\footnotetext{
${ }^{2}$ The notion of an adapted stochastic process $\left\{\xi_{t}\right\}_{t \geq 0}$ implies that each random variable $\xi_{t}$ is $\mathcal{F}_{t^{-}}$ measurable and, therefore, can only depend on incomes $e_{\tau}$ observed during periods $\tau \leq t$. As a notational convention, inequalities involving random variables are understood to hold in the $\mathbb{P}$-almost sure sense without explicit notice.
} 
The initial value $b_{0} \geq 0$ is given historically. ${ }^{3}$ Structurally, the contracts supplied by banks can be interpreted as inside money (see Gale (1973) for an interpretation of negative inside money).

\section{Consumer demand}

The young choose $\left(c^{y}, c^{o}, h\right)$ to maximize their expected lifetime utility based on some von-Neumann Morgenstern utility function $U$, which is additively separable over time, i.e.,

$$
U\left(c^{y}, c^{o}, h\right)=u\left(c^{y}\right)+v\left(c^{o}, h\right) .
$$

The function $u$ is taken to be of the isoelastic form

$$
u(c)=\frac{c^{1-\alpha}}{1-\alpha}, \quad \alpha>0
$$

with the usual interpretation that $u(c)=\log c$ if $\alpha=1$. Second period utility $v$ is the composition of $u$ and an aggregator function $g: \mathbb{R}_{++}^{2} \rightarrow \mathbb{R}_{+}$which aggregates durable and non-durable consumption to a composite commodity $g\left(c_{t}, h_{t}\right)$. Following Bajari, Chan, Krueger \& Miller (2010) or Lustig \& Nieuwerburgh (2005), we use a CES aggregator

$$
g(c, h)=\left[\beta c^{\rho}+(1-\beta) h^{\rho}\right]^{\frac{1}{\rho}}, 0<\beta<1, \rho<1 .
$$

The young discount second-period utility by $\gamma>0$ and thus $v$ takes the form

$$
v(c, h)=\gamma u(g(c, h))=\gamma \frac{\left[\beta c^{\rho}+(1-\beta) h^{\rho}\right]^{\frac{1-\alpha}{\rho}}}{1-\alpha} .
$$

If $\rho=0, v$ is Cobb-Douglas while it is additively separable in housing and consumption if $\rho=1-\alpha$.

Given $p_{t}>0, R_{t}>0$, and $p_{t+1}>0$, the budget constraints are

$$
c^{y}=e_{t}^{y}+b-p_{t} h \quad \text { and } \quad c^{o}=e_{t+1}^{o}-R_{t} b+\left(p_{t+1}-\kappa\right) h .
$$

where $b$ and $h$ are the loan demand and housing investment respectively. Let $\mathbb{E}_{t}[\cdot]:=$ $\mathbb{E}\left[\cdot \mid \mathcal{F}_{t}\right]$ denote the expectations operator conditional on the information available at time $t$. Using (5), the young consumers' objective function at time $t$ is

$$
V_{t}(b, h):=\mathbb{E}_{t}\left[U\left(e_{t}^{y}+b-p_{t} h, e_{t+1}^{o}-R_{t} b+\left(p_{t+1}-\kappa\right) h, h\right)\right] .
$$

Note that the housing price $p_{t+1}$ is the only potential source of uncertainty. The consumers' decision problem reads

$$
\max _{b, h}\left\{V_{t}(b, h) \mid p_{t} h \leq e_{t}^{y}+b, e_{t+1}^{o}-b R_{t}+h\left(p_{t+1}-\kappa\right) \geq 0, h \geq 0\right\} .
$$

\footnotetext{
${ }^{3}$ An initial value $b_{0}<0$ would correspond to the case where banks take deposits. As our interest is on banks granting mortgage loans we do not study this case.
} 
Note that no sign restriction on $b$ is imposed at the individual level.

\section{Equilibrium.}

The following definition of equilibrium reconciles market clearing and individual optimality under rational expectations.

\section{Definition 1}

Let incomes satisfy Assumption 1. Given an initial credit volume $b_{0} \geq 0$, an equilibrium is an adapted stochastic process $\left\{b_{t}, h_{t}, R_{t}, p_{t}\right\}_{t \geq 0}$, which satisfies $p_{t}>0, R_{t}>0$, and the following conditions for each $t \geq 0$ :

(i) The decision $\left(b_{t}, h_{t}\right)$ solves (7) given prices and incomes.

(ii) Markets clear, i.e., $h_{t}=1$ and $b_{t}$ evolves according to (1).

Note that Walras' law implies consumption good market clearing, i.e, $c_{t}^{y}+c_{t}^{o}=e_{t}^{y}+e_{t}^{o}-\kappa$ for all $t \geq 0$.

\section{Recursive Equilibrium Structure}

\section{Predictable housing prices}

To study the existence and dynamic properties of equilibria, we consider the case where housing prices are predictable, i.e., their realization can be predicted one period in advance. This assumption enables us to obtain an analytically tractable equilibrium. It imposes no restrictions in the case when incomes are deterministic. Formally, we assume that the housing price $p_{t+1}$ is $\mathcal{F}_{t}$-measurable. Under this hypothesis, the young at time $t$ solve a decision problem under certainty such that the expectations operator in (6) can be dropped.

\section{Recursive equilibrium}

As a first step, we unveil the forward-recursive structure of equilibrium and the state dynamics of the model. Essentially, we will show that the dynamics is driven by the evolution of the variable

$$
q_{t}:=p_{t}-b_{t}, \quad t \geq 0,
$$

which, we show, can be interpreted as the fundamental housing price. We focus on equilibria where $q_{t}>0$ for all $t \geq 0$ such that loans are fully backed by housing values. This may be interpreted as a collateral constraint. Since housing prices are predictable and banks offer riskless interest rates, the process $\left\{q_{t}\right\}_{t \geq 0}$ is predictable as well.

Since no sign-restriction is imposed on $b$ at the individual level, the first order conditions of the young consumers' decision problem (7) must be satisfied in equilibrium. 
Exploiting this, (2), and (8), the following Euler equations have to hold for each period $t \geq 0$ :

$$
\begin{aligned}
u^{\prime}\left(e_{t}^{y}-q_{t}\right) & =R_{t} v_{c}\left(e_{t+1}^{o}-\kappa+q_{t+1}, 1\right) \\
p_{t} u^{\prime}\left(e_{t}^{y}-q_{t}\right) & =\left(p_{t+1}-\kappa\right) v_{c}\left(e_{t+1}^{o}-\kappa+q_{t+1}, 1\right)+v_{h}\left(e_{t+1}^{o}-\kappa+q_{t+1}, 1\right) .
\end{aligned}
$$

From (9a) and (9b) we infer that the interest factor $R_{t}$ must be higher than the return from housing $\left(p_{t+1}-\kappa\right) / p_{t}$. This is because the old derive utility from housing. Given $e=\left(e^{y}, e^{o}\right) \in \mathcal{E}$, let $F(\cdot, \cdot ; e):\left(\kappa-e^{0}, \infty\right) \times\left(-\infty, e^{y}\right) \rightarrow \mathbb{R}$ where

$$
F\left(q_{1}, q ; e\right):=q u^{\prime}\left(e^{y}-q\right)-v_{c}\left(e^{o}-\kappa+q_{1}, 1\right)\left(q_{1}-\kappa\right)-v_{h}\left(e^{o}-\kappa+q_{1}, 1\right) .
$$

Then, adding (9a) and (9b) using (1) and (8) gives the following equilibrium condition

$$
F\left(q_{t+1}, q_{t} ; e_{t}\right)=0
$$

which has to hold at each time $t \geq 0$. Condition (11) determines the value $q_{t+1}$ implicitly as a function of $q_{t}$ and $e_{t}$. The following result states necessary and sufficient conditions under which a unique solution to (11) can be determined.

\section{Lemma 1}

Suppose $\rho \geq 0$ and $\alpha<1$. Then, for each $e=\left(e^{y}, e^{o}\right) \in \mathcal{E}$ and $q<e^{y}$ there exists a unique value $q_{1}>\kappa-e^{o}$, which satisfies $F\left(q_{1}, q ; e\right)=0$.

Lemma 1 permits to define a map $f(\cdot ; e):\left(-\infty, e^{y}\right) \rightarrow\left(\kappa-e^{o}, \infty\right)$ which determines the unique zero of $F(\cdot, q ; e)=0$ for each $q<e^{y}{ }^{4}$ Thus, whenever $q_{t}<e_{t}^{y}$, the solution to $(11)$ can be written as

$$
q_{t+1}=f\left(q_{t} ; e_{t}\right)
$$

The next result establishes properties of $f$.

\section{Lemma 2}

Suppose $\rho \geq 0$ and $\alpha<1$. Then, for each $e=\left(e^{y}, e^{0}\right) \in \mathcal{E}$ the map $f=f(\cdot ; e)$ is continuously differentiable with derivative $f^{\prime}(q)>0$ for all $q<e^{y}$.

Using the result from (12) in (9a) and (9b), the equilibrium interest factor and next period's (expected) housing price are determined from $e_{t} \in \mathcal{E}, q_{t}<e_{t}^{y}$ and $p_{t}$ by

$$
\begin{aligned}
R_{t}=\mathcal{R}\left(q_{t} ; e_{t}\right) & :=\frac{u^{\prime}\left(e_{t}^{y}-q_{t}\right)}{v_{c}\left(e_{t+1}^{o}-\kappa+f\left(q_{t} ; e_{t}^{y}\right), 1\right)} \\
p_{t+1}=\mathcal{P}\left(p_{t}, q_{t} ; e_{t}\right) & :=\mathcal{R}\left(q_{t}, e_{t}\right) p_{t}+\kappa-\frac{v_{h}\left(e_{t+1}^{o}-\kappa+f\left(q_{t} ; e_{t}\right), 1\right)}{v_{c}\left(e_{t+1}^{o}-\kappa+f\left(q_{t} ; e_{t}\right), 1\right)}
\end{aligned}
$$

\footnotetext{
${ }^{4}$ The restrictions $\rho \geq 0$ and $\alpha<1$ are necessary and sufficient for $\lim _{c \rightarrow \infty} v_{c}(c, 1) c=\infty$ which is crucial for existence of a solution to (11) for arbitrary $q_{t}$ and $e_{t}$. Although the restriction $\alpha<1$ excludes a logarithmic function $u$ used in Bajari, Chan, Krueger \& Miller (2010), this case can be approximated as the limiting case $\alpha \rightarrow 1$ in our setup.
} 
while loans $b_{t+1}$ follow from (1). Equation (13) equates the equilibrium interest factor to the intertemporal marginal rate of substitution in consumption. One also infers from (14) that the equilibrium housing return $\left(p_{t+1}-\kappa\right) / p_{t}$ must be smaller than $R_{t}$ due to the positive marginal rate of substitution between housing and second period consumption. Also, note that $q_{t}>0$ ensures that $p_{t}>0$ by (8).

\section{Housing Price Dynamics}

\section{Dynamics under constant incomes}

Based on the previous results, we are now ready to study the dynamics of the model and establish conditions for the existence of equilibrium. To this end, note that (12) does not yet define a dynamical system because we have not determined a suitable state space $\mathbb{Q}$ on which the dynamics can live. While this section studies the equilibrium dynamics under constant first-period income, the next section will extend this to the case where first-period income is random. Let us first fix $e_{t} \equiv e=\left(e^{y}, e^{o}\right) \in \mathcal{E}$ for all $t \geq 0$. For notational convenience, the dependence of variables and functions on $e$ will be suppressed. We seek to determine an interval $\mathbb{Q} \subset\left[0, e^{y}\right)$ which is self-supporting for the map $f=f(\cdot ; e)$, i.e., $f(q) \in \mathbb{Q}$ for all $q \in \mathbb{Q}$. By the properties of $f$ established in Lemma 2, fixed points, i.e., values $q$ which satisfy $q=f(q)$, are natural boundary points of intervals which are self-supporting under $f$. Thus, we begin by studying fixed points of $f$ and their properties. Since $f$ maps $\left(-\infty, e^{y}\right)$ into $\left(\kappa-e^{o}, \infty\right)$, it is clear that any such fixed point must lie in the open interval $\left(\kappa-e^{o}, e^{y}\right)$. Therefore, a necessary precondition for fixed points to exist is $e^{y}+e^{o}>\kappa$. This condition simply says that the resources available in each period are large enough to cover housing costs. Below, we even require a stronger condition that $e^{o}>\kappa$. It follows from (10) that fixed-points of $f$ obtain as zeros of the map $G:\left(\kappa-e^{o}, e^{y}\right) \rightarrow \mathbb{R}$ where

$$
\begin{aligned}
G(q) & :=F(q, q ; e) \\
& =q u^{\prime}\left(e^{y}-q\right)-v_{c}\left(e^{o}-\kappa+q, 1\right)(q-\kappa)-v_{h}\left(e^{o}-\kappa+q, 1\right) .
\end{aligned}
$$

The following result states properties of the map $G$.

\section{Lemma 3}

Suppose $\rho \geq 0$ and $\alpha<1$. Then, for each $e=\left(e^{y}, e^{0}\right) \in \mathcal{E}$ satisfying $e^{y}+e^{o}>\kappa$ the map $G=G(\cdot ; e)$ is a strictly convex function and the derivative satisfies the boundary behavior $\lim _{q \rightarrow e^{y}} G^{\prime}(q)=-\lim _{q \rightarrow \kappa-e^{o}} G^{\prime}(q)=\infty$.

A consequence of the lemma is the existence of a unique value $q_{\min } \in\left(\kappa-e^{o}, e^{y}\right)$ at which $G^{\prime}\left(q_{\min }\right)=0$ and $G$ attains its global minimum. Based on this insight, the next result states conditions for fixed points to exist and characterizes their properties. 


\section{Lemma 4}

Suppose $\rho \geq 0$ and $\alpha<1$. Let incomes $e=\left(e^{y}, e^{0}\right) \in \mathcal{E}$ be given and assume that $e^{y}+e^{o}>\kappa$ and $G\left(q_{\min }\right)<0$. Then,

(i) The map $f$ has precisely two fixed points $\bar{q} \in\left(\kappa-e^{o}, q_{\min }\right)$ and $\overline{\bar{q}} \in\left(q_{\min }, e^{y}\right)$.

(ii) The fixed point $\overline{\bar{q}}$ is locally unstable while $\bar{q}$ is asymptotically stable. Moreover, $f(q)>q$ for all $q \in(-\infty, \bar{q}) \cup\left(\overline{\bar{q}}, e^{y}\right)$ and $f(q)<q$ for all $q \in(\bar{q}, \overline{\bar{q}})$.

Setting aside the non-generic case where $G\left(q_{\min }\right)=0$, the boundary behavior of $G$ implies that the condition $G\left(q_{\min }\right)<0$ is not only sufficient but also necessary for fixed points to exist. Moreover, it can be shown that $G\left(q_{\min }\right)>0$ would imply $f(q ; e)>q$ for all $q$. In this case, for any $q_{0}<e^{y}$ a repeated iteration of the forward-recursion (12) would produce a value $q_{t}>e^{y}$ after finitely many periods $t \geq 1$. Thus, $G\left(q_{\min }\right)<0$ is also a necessary condition for the dynamics to be viable. The finding from Lemma 4 is illustrated in the following figures which depict the map $f$ and the fixed point map $G$. Note that the zeros of $G$ in Figure 1(b) correspond to intersections of (the graph of) $f$ with the principal diagonal in Figure 1(a).

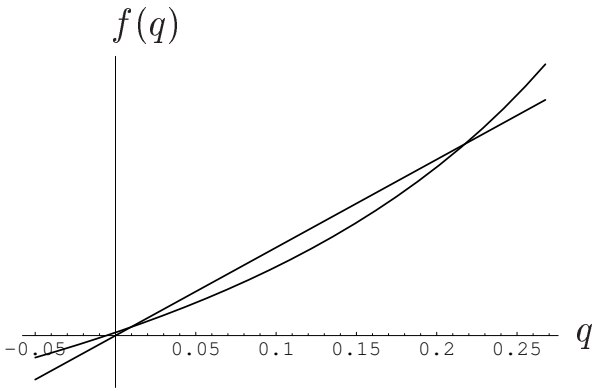

(a) The map $f$

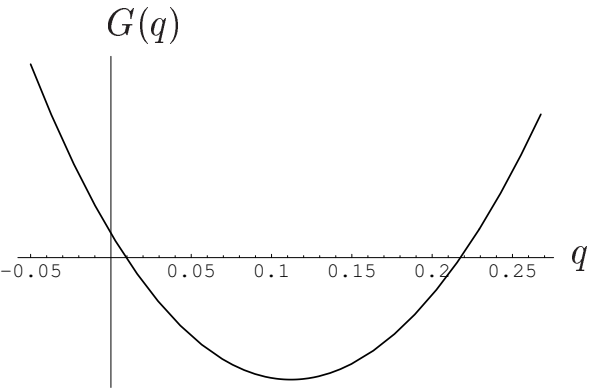

(b) Fixed point map $G$

Figure 1: Time-one map of state dynamics

Lemma 4(ii) reveals that the set $(-\infty, \bar{q}]$ is self-supporting under $f$. Thus, the map $f$ restricted to this set becomes the time-one map of a one-dimensional dynamical system in discrete time. ${ }^{5}$ Moreover, for any initial value $q_{0} \in(-\infty, \overline{\bar{q}})$ the sequence $\left\{q_{t}\right\}_{t \geq 0}$ defined recursively as $q_{t+1}=f\left(q_{t}\right), t \geq 0$ converges monotonically to $\bar{q}$. By contrast, for any initial value $q_{0} \in\left(\overline{\bar{q}}, e^{y}\right)$ the forward-recursion $q_{t+1}=f\left(q_{t}\right)$ exceeds $e^{y}$ after finitely many periods. Thus, the forward dynamics is well-defined if and only if $q_{0} \in(-\infty, \overline{\bar{q}}]$. In the sequel, we will exclude $\overline{\bar{q}}$ from the state space in order to rule out degenerate equilibria. ${ }^{6}$ To ensure $q_{t} \geq 0$, we assume that the smaller fixed point satisfies $\bar{q}>0$. Then, Lemma $4($ ii) in conjunction with Lemma 2 imply that the interval $\mathbb{Q}:=[0, \overline{\bar{q}})$ is

\footnotetext{
${ }^{5}$ For convenience, we denote the restriction of $f$ to a subset $\mathbb{Q} \subset\left(-\infty, e^{y}\right)$ by $f$ as well.

${ }^{6}$ In the stochastic case to be studied in the next section, this imposes no additional restriction regarding the long run behavior of the system.
} 
self-supporting under $f$ as well. Thus, restricting $f$ to $\mathbb{Q}$ defines a discrete dynamical system which governs the evolution of the variable $q_{t} \in \mathbb{Q}$ over time. The long-run behavior is now characterized in the following result, which requires in addition $e^{o}>\kappa$.

\section{Lemma 5}

Suppose $\rho \geq 0$ and $\alpha<1$. Let incomes $e=\left(e^{y}, e^{0}\right) \in \mathcal{E}$ be given and assume that $e^{o}>\kappa$ and $G\left(q_{\min }\right)<0<\bar{q}$. Then,

(i) The restricted map $f: \mathbb{Q} \rightarrow \mathbb{Q}$ has $\bar{q}$ as its unique fixed point.

(ii) This fixed point is globally stable and for each $q_{0} \in \mathbb{Q}$ the sequence $\left\{q_{t}\right\}_{t \geq 0}$ defined recursively as $q_{t+1}=f\left(q_{t} ; e^{y}\right), t \geq 0$ converges monotonically to $\bar{q}$.

In addition to the parameter restrictions $\rho \geq 0$ and $\alpha<1$, the previous findings show that the main restrictions needed for the dynamics to be well-defined are that $G\left(q_{\min }\right)<$ 0 and, in addition, $\bar{q}>0$. The latter is necessary and sufficient for the dynamics to live in a subset of $\mathbb{R}_{++}$and satisfied if and only if $G^{\prime}(0)<0<G(0)$. Using (4) in (15), direct computations give

$$
\begin{aligned}
& G(0)>0 \quad \Leftrightarrow \quad \kappa>\frac{v_{h}\left(e^{o}-\kappa, 1\right)}{v_{c}\left(e^{o}-\kappa, 1\right)}=\frac{1-\beta}{\beta}\left[e^{o}-\kappa\right]^{1-\rho} \\
& G^{\prime}(0)<0 \quad \Leftrightarrow \quad u^{\prime}\left(e^{y}\right)<(1-\alpha) v_{c}\left(e^{o}-\kappa, 1\right)-e^{o} v_{c c}\left(e^{o}-\kappa, 1\right) .
\end{aligned}
$$

Condition (16a) shows that $G(0)>0$ is violated for $\kappa=0$ and, therefore, requires housing costs to exceed a critical level which depends on $e^{o} .^{7}$ As this condition is independent of first-period income, the value $e^{y}$ can always be chosen sufficiently large to satisfy the second condition $(16 \mathrm{~b})$. Notice, however, that the condition $G\left(q_{\min }\right)<0$ depends on these choices as well. Given that $q_{\min }$ is only implicitly defined by $G^{\prime}(q)=$ 0 , it is difficult to fully characterize the underlying parameter sets. The numerical simulation of the following section, however, shows that all three conditions are satisfied for a broad range of economically reasonable parameterizations.

\section{Equilibrium under constant incomes}

We are now in a position to characterize the complete equilibrium dynamics for a fixed income process $e_{t} \equiv e=\left(e^{y}, e^{o}\right) \in \mathcal{E}$ for which all hypotheses of Lemma 5 are satisfied. Fix an initial value $\left(p_{0}, b_{0}\right)$ which satisfies $b_{0} \geq 0, p_{0}>0$, and $q_{0}=p_{0}-b_{0} \in \mathbb{Q}$. Then, combining our previous results with (1), (8), (13), and (14) one obtains the following

\footnotetext{
${ }^{7}$ As argued above, the housing return must be lower than the interest factor at equilibrium. If $\kappa=0$, then $p_{t+1} / p_{t}<b_{t+1} / b_{t}=R_{t}$. This implies that the loan volume grows faster than the housing price and thus, within finite time the old will not be able to repay their debt. A similar conclusion holds in a modified version of our model where housing is replaced by a Lucas' tree traded at price $p_{t}$, which yields a positive dividend in each period. The scenario with zero dividend payments corresponds to the case with fiat money where the economy converges to an autarky steady state.
} 
system which governs the evolution of all equilibrium variables:

$$
\begin{aligned}
q_{t+1} & =f\left(q_{t} ; e\right) \\
b_{t+1} & =\mathcal{R}\left(q_{t} ; e\right) b_{t} \\
p_{t+1} & =f\left(q_{t} ; e\right)+\mathcal{R}\left(q_{t} ; e\right) b_{t} .
\end{aligned}
$$

The dynamics (17a) of $q_{t}$ is decoupled from the other two variables and converge monotonically to a unique steady state $\bar{q}$ by Lemma 5 . Moreover, this process defines the induced consumption allocation as

$$
\begin{aligned}
c_{t}^{y} & =e_{t}^{y}-q_{t} \\
c_{t}^{o} & =e_{t}^{0}-\kappa+q_{t} .
\end{aligned}
$$

It is evident from (17b) and (17c) that the qualitative long-run dynamic behavior of the credit volume $b_{t}$ and housing prices $p_{t}$ depend on the steady state interest factor $\mathcal{R}(\bar{q} ; e)$. If $\mathcal{R}(\bar{q} ; e)<1$, the credit volume asymptotically converges to zero while by (8) prices converge to $\bar{p}=\bar{q}$. Conversely, if $\mathcal{R}(\bar{q} ; e)>1$ and $b_{0}>0$, both the credit volume and housing prices grow without bound and converge to infinity. Notice, however, that the equilibrium dynamics is well-defined in either case. The following final theorem of this section summarizes these insights and establishes the existence and properties of equilibrium.

\section{Theorem 1}

Suppose $\rho \geq 0$ and $\alpha<1$. Let incomes $e=\left(e^{y}, e^{0}\right) \in \mathcal{E}$ be given and assume that $e^{o}>\kappa$ and $G\left(q_{\min }\right)<0<\bar{q}$. Then,

(i) Each $p_{0}>0$ and $b_{0} \geq 0$ for which $p_{0}-b_{0} \in \mathbb{Q}$ defines an equilibrium where the evolution of the equilibrium variables follows $(17 \mathrm{a}-c)$ and $\lim _{t \rightarrow \infty} q_{t}=\bar{q}$.

(ii) If $b_{0}>0$ and $\mathcal{R}(\bar{q} ; e)>1$, then $\lim _{t \rightarrow \infty} p_{t}=\lim _{t \rightarrow \infty} b_{t}=\infty$.

(iii) If $b_{0}=0$ or $\mathcal{R}(\bar{q} ; e)<1$, then $\lim _{t \rightarrow \infty} p_{t}=\bar{q}$ while $\lim _{t \rightarrow \infty} b_{t}=0$.

\section{$5 \quad$ Housing Booms and Busts}

\section{Dynamics under random incomes}

We now analyze the case where incomes fluctuate randomly over time. For ease of exposition, we will confine attention to the case where only first-period incomes change over time while second-period incomes are assumed to be constant. Thus, assume as in the previous section that $e_{t}^{o} \equiv e^{o}>\kappa$ while $e_{t}^{y}$ fluctuates over time taking values in the set $\mathcal{E}^{y}:=\left[e_{\min }^{y}, e_{\text {max }}^{y}\right] \subset \mathbb{R}_{++}$. In the sequel, we will therefore drop the argument $e^{o}$ writing e.g. $f\left(q ; e^{y}\right)$ instead of $f\left(q ; e^{y}, e^{o}\right)$. Consider first how the process $\left\{q_{t}\right\}_{t \geq 0}$ 
defined in (8) evolves over time. It is clear from (12) that this process is well-defined if and only if $q_{t}<e_{t}^{y}$ for all $t \geq 0 \mathbb{P}$-a.s., i.e., $q_{t}<e_{\min }^{y}$ for all $t \geq 0 \mathbb{P}$-a.s. Suppose this can be satisfied. Then, the forward-recursive structure of the model is well-defined and generated by randomly mixing the family of mappings $\left(f \cdot ; e^{y}\right)_{e^{y} \in \mathcal{E}^{y}}$. That is, given $q_{t}$, the value $e_{t}^{y} \in \mathcal{E}$ realized at time $t$ 'selects' a particular map $f\left(\cdot ; e_{t}^{y}\right)$ which determines the next value $q_{t+1}=f\left(q_{t} ; e_{t}^{y}\right)$. For this forward-recursion to be well-defined, we seek to determine a stable interval $\mathbb{Q} \subset\left[0, e_{\text {min }}^{y}\right)$ which is self supporting under the family $\left(f \cdot ; e^{y}\right)_{e^{y} \in \mathcal{E}^{y}}$, i.e., $q \in \mathbb{Q}$ implies $f\left(q ; e^{y}\right) \in \mathbb{Q}$ for all $e^{y} \in \mathcal{E}^{y}$.

While the underlying construction principle is the same as in the previous section, the present case must incorporate that the map $f$ and its fixed points vary with the income process. Let us assume that the hypotheses of Lemma 5 are satisfied for all $e^{y} \in \mathcal{E}^{y}$. Then, each map $f\left(\cdot ; e^{y}\right)$ has precisely two fixed points in $\left(0, e^{y}\right)$ which we denote by $\bar{q}\left(e^{y}\right)$ and $\bar{q}\left(e^{y}\right)$, respectively. The next result describes how these fixed points vary with income.

\section{Lemma 6}

Let the hypotheses of Lemma 5 be satisfied for each $e^{y} \in \mathcal{E}^{y}$. Then,

(i) For each $q>0$ the map $e^{y} \mapsto f\left(q ; e^{y}\right)$ is continuously differentiable (on the interior of $\left.\mathcal{E}^{y}\right)$ and strictly decreasing.

(ii) The fixed point maps $e^{y} \mapsto \bar{q}\left(e^{y}\right)$ and $e^{y} \mapsto \overline{\bar{q}}\left(e^{y}\right)$ are both continuously differentiable. Moreover, $\bar{q}(\cdot)$ is strictly decreasing while $\overline{\bar{q}}(\cdot)$ is strictly increasing.

Using the previous result, define

$$
\begin{aligned}
& \bar{q}_{\min }:=\min _{e^{y} \in \mathcal{E}^{y}}\left\{\bar{q}\left(e^{y}\right)\right\}=\bar{q}\left(e_{\max }^{y}\right) \\
& \bar{q}_{\max }:=\max _{e^{y} \in \mathcal{E}^{y}}\left\{\bar{q}\left(e^{y}\right)\right\}=\bar{q}\left(e_{\min }^{y}\right) \\
& \overline{\bar{q}}_{\min }:=\min _{e^{y} \in \mathcal{E}^{y}}\left\{\overline{\bar{q}}\left(e^{y}\right)\right\}=\overline{\bar{q}}\left(e_{\min }^{y}\right) .
\end{aligned}
$$

Note that the values defined in $(19 \mathrm{a}-\mathrm{c})$ satisfy $0<\bar{q}_{\min }<\bar{q}_{\max }<\overline{\bar{q}}_{\min }$. Thus, defining $\overline{\mathbb{Q}}:=\left[\bar{q}_{\min }, \bar{q}_{\max }\right]$ and $\mathbb{Q}:=\left[0, \overline{\bar{q}}_{\text {min }}\right)$ we have the inclusions $\emptyset \neq \overline{\mathbb{Q}} \varsubsetneqq \mathbb{Q}$. The following result essentially extends Lemma 5 to the more general stochastic case.

\section{Lemma 7}

Let the hypotheses of Lemma 5 be satisfied for each $e^{y} \in \mathcal{E}^{y}$. Then,

(i) Both intervals $\overline{\mathbb{Q}}$ and $\mathbb{Q}$ are self-supporting for the family $\left(f \cdot: e^{y}\right)_{e^{y} \in \mathcal{E}^{y}}$.

(ii) For each $q_{0} \in \mathbb{Q}$, the dynamics generated by randomly mixing $\left(f \cdot ; e^{y}\right)_{e^{y} \in \mathcal{E}^{y}}$ converge to the set $\overline{\mathbb{Q}} \mathbb{P}-$ a.s.. 
It follows from Lemma 7 that asymptotically, the process $\left\{q_{t}\right\}_{t \geq 0}$ will take values in the set $\overline{\mathbb{Q}}$. Thus, if $\left\{e_{t}^{y}\right\}_{t \geq 0}$ is sufficiently regular, e.g., follows a Markov process, standard results from the literature (cf. Brock \& Mirman (1972) and Wang (1993)) imply the existence of a unique invariant distribution supported on $\overline{\mathbb{Q}}$ which governs the longrun probabilistic behavior of the process $\left\{q_{t}\right\}_{t \geq 0}$. In particular, this process will be asymptotically stationary. ${ }^{8}$ Figure 2 illustrates the finding from Lemma 7 for the case with two shocks where $e_{t}^{y} \in\left\{e_{\min }^{y}, e_{\max }^{y}\right\}$ for all $t$.

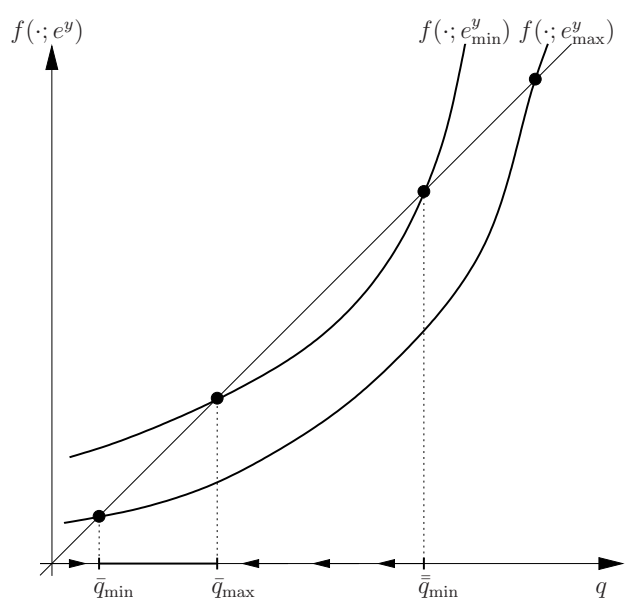

Figure 2: Time-one maps generating the dynamics under two shocks

\section{Equilibrium under random incomes}

Based on the previous result, the following theorem generalizes the existence result from Theorem 1(i) to the case with stochastic first-period incomes. Note that Theorem 1(i) obtains as a special case where $e_{\min }^{y}=e_{\max }^{y}=e^{y}$.

\section{Theorem 2}

Let the hypotheses of Lemma 5 be satisfied for each $e^{y} \in \mathcal{E}^{y}$. Then, each $p_{0}>0$ and $b_{0} \geq 0$ for which $q_{0}:=p_{0}-b_{0} \in \mathbb{Q}$ defines an equilibrium process generated by (12), (13), and (14).

Since we are interested in the long-run properties of equilibrium, we can confine attention to the set $\overline{\mathbb{Q}}$ by virtue of Lemma $7(\mathrm{ii})$. Analogously to the previous section, the sign of the interest rate is crucial for the long-run behavior of equilibrium housing prices and the credit volume. The following result characterizes how the interest factor changes along with the shocks and the value of $q$.

\footnotetext{
${ }^{8}$ Lemma 4(ii) and the definitions $(19 \mathrm{a}-\mathrm{c})$ imply that the family $f=\left(f \cdot: e^{y}\right)_{e^{y} \in \mathcal{E}}$ restricted to the interval $\mathbb{Q}$ possesses a stable fixed-point configuration in the sense of Brock \& Mirman (1972). Thus, the assertion follows from their results, see also Wang (1993).
} 


\section{Lemma 8}

Let the hypotheses of Lemma 5 be satisfied for each $e^{y} \in \mathcal{E}^{y}$. Then, the map $\mathcal{R}$ defined in (13) is continuously differentiable with partial derivatives $\mathcal{R}_{e^{y}}\left(q ; e^{y}\right)<0<\mathcal{R}_{q}\left(q ; e^{y}\right)$ for all $e^{y}>0$ and $q<e^{y}$.

Let

$$
\begin{aligned}
& R_{\text {min }}:=\min \left\{\mathcal{R}\left(q ; e^{y}\right) \mid q \in \overline{\mathbb{Q}}, e^{y} \in \mathcal{E}\right\}=\mathcal{R}\left(\bar{q}_{\text {min }} ; e_{\text {max }}^{y}\right) \\
& R_{\text {max }}:=\max \left\{\mathcal{R}\left(q ; e^{y}\right) \mid q \in \overline{\mathbb{Q}}, e^{y} \in \mathcal{E}\right\}=\mathcal{R}\left(\bar{q}_{\text {max }} ; e_{\text {min }}^{y}\right)
\end{aligned}
$$

We now have the following result which extends the characterization of equilibrium from Theorem 1(ii) and (iii) to the general stochastic case.

\section{Theorem 3}

Let the hypotheses of Lemma 5 be satisfied for each $e^{y} \in \mathcal{E}^{y}$. Then,

(i) If $b_{0}>0$ and $R_{\min }>1$, then $\lim _{t \rightarrow \infty} p_{t}=\lim _{t \rightarrow \infty} b_{t}=\infty, \mathbb{P}-$ a.s.

(ii) If $b_{0}=0$ or $R_{\max }<1$, then $\lim _{t \rightarrow \infty} b_{t}=0 \mathbb{P}$-a.s. while $\lim _{t \rightarrow \infty}\left|p_{t}-q_{t}\right|=0 \mathbb{P}$-a.s.

Theorem 3 shows that the long-run behavior of housing prices and the credit volume is either expansive (i) or stationary (ii). We observe that if $b_{0}=0$, i.e., in the absence of a banking sector, the housing price coincides with the process $\left\{q_{t}\right\}_{t \geq 0}$ which is stationary and well-behaved. Thus, any potential non-stationarity in housing prices is exclusively due to the banking sector. Excluding the non-generic cases of either $R_{\min }=1$ or $R_{\max }=1$, recurrent housing booms and busts can emerge only if $R_{\min }<1<R_{\max }$.

The mechanism for booms and busts

To illustrate a mechanism that generates booms and busts of housing prices, consider the simplest case where $e^{y}$ takes two values $e_{\min }^{y}$ and $e_{\max }^{y}$ with positive probability. Let $b_{0}>0$ and $R_{\min }<1<R_{\max }$. Suppose that incomes initially take the lower value $e_{t}^{y}=e_{\min }^{y}$. Then, the dynamics generated by the map $f\left(\cdot ; e_{\min }^{y}\right)$ start converging to the associated steady state $\bar{q}\left(e_{\min }^{y}\right)=\bar{q}_{\max }$ and we have $R_{t}>1$ for $t$ sufficiently large as $\mathcal{R}\left(\bar{q}_{\max }, e_{\min }^{y}\right)=R_{\max }>1$. By (1), the credit volume starts to expand and so do housing prices while their difference $q_{t}$ is stationary. Intuitively, the low first period income increases the need for consumption smoothing and the demand for credit, for which the young are willing to pay a high interest rate. Although the supply of credit expands over time as well, this is absorbed by a corresponding higher demand due to increasing housing prices. Thus, we see that as long as the low income regime prevails, both housing price and credit volume increase whereas their difference converges to $\bar{q}_{\max }$.

Now, suppose that at some time $\tilde{t}>0$, incomes switch to the higher value $e_{\max }^{y}$. The corresponding dynamics is now generated by the map $f\left(\cdot ; e_{\max }^{y}\right)$ which has $\bar{q}_{\min }$ as its unique steady state to which the variable $q_{t}$ starts converging. For sufficiently large 
$t>\tilde{t}$, we will have $R_{t}<1$ implying that both the credit volume and housing price will contract. Although this induces a -perfectly foreseen- capital loss, the demand for housing is still positive as it yields utility.

Combining these observations, it is clear that under a random income process, the system will alternate between an expansionary regime and a contractive regime. These changes are most profound if $\mathcal{R}\left(q ; e_{\text {min }}^{y}\right)>1$ and $\mathcal{R}\left(q ; e_{\text {max }}^{y}\right)<1$ for all $q \in \overline{\mathbb{Q}}$. The first requirement is equivalent to $\mathcal{R}\left(\bar{q}_{\min }, e_{\text {min }}^{y}\right)>1$ and implies that the credit volume starts expanding immediately when $e_{t}=e_{\min }^{y}$. The second condition is equivalent to $\mathcal{R}\left(\bar{q}_{\text {max }}, e_{\text {max }}^{y}\right)<1$ and implies that the credit volume starts contracting immediately when $e_{t}=e_{\min }^{y}$. Now if the income process is persistent, then long periods of credit expansion will follow long periods of credit contraction. This mechanism offers a potential to generate large movements in housing prices simply due to persistent income changes.

The previous mechanism straightforwardly generalizes to the case where incomes are continuously distributed on the interval $\left[e_{\min }^{y}, e_{\max }^{y}\right]$ as long as the dynamics alternates between the expansive regime $\left\{(q, e) \in \overline{\mathbb{Q}} \times \mathcal{E}^{y} \mid \mathcal{R}(q ; e)>1\right\}$ and the contractive regime $\left\{(q, e) \in \overline{\mathbb{Q}} \times \mathcal{E}^{y} \mid \mathcal{R}(q ; e)<1\right\}$.

\section{Bubbles and fundamental housing prices}

It is worthwhile to relate our previous results to the emergence of a bubble which is widely discussed in the literature. In these models the notion of bubbles corresponds to an intrinsically valueless asset that is traded at a positive price. In our model, the credit volume supplied by the banking sector is not backed by any resources and satisfies this definition. Consequently, the bubble-less equilibrium in our economy corresponds to the initial choice $b_{0}=0$ which implies $b_{t} \equiv 0$ and $p_{t}=q_{t}$ for all $t$. Therefore, we shall call $q_{t}$ the fundamental housing price. We know from our previous results that for any $q_{0}>0$, the fundamental housing price process $\left\{q_{t}\right\}_{t \geq 0}$ is well-behaved and converges to a unique stable interval $\left[q_{\min }, q_{\max }\right] \subset\left(0, e_{\min }^{y}\right)$ which shrinks to a point $\bar{q}$ if incomes are deterministic.

Under this interpretation, it follows directly from (8) that for an arbitrary $b_{0} \geq 0$, the equilibrium housing price $p_{t}$ may be written as the sum of its fundamental value $q_{t}$ and the bubbly component $b_{t}$. Given a fixed initial fundamental price $q_{0}$, any injection of credit $b_{0}>0$ therefore merely increases the bubbly component of the housing price. With the initial fundamental price $q_{0}$ unchanged, one also observes from (17a) and $(18 \mathrm{a}, \mathrm{b})$ that the resulting consumption allocation is not affected by the presence of a bubble. Thus, any injection of credit is fully neutral with respect to consumer welfare. Housing investment is in part financed by first period income and selling revenue exceeds the loan repayment. Thus, at equilibrium there is an effective transfer of resources from the young to old. If incomes alternate between the expansive and contractive state, large movements in housing prices occur which can almost exclusively be attributed to the bubbly component with the fundamental price being bounded by the income process. 


\section{A Quantitative Example}

We employ numerical simulations to show that the boom-bust scenario studied previously occurs under reasonable parameter choices and the switch between the two regimes is triggered by relatively small income changes.

\section{Parameters}

Bajari, Chan, Krueger \& Miller (2010) use a logarithmic function $u$ which we approximate in our setup by choosing $\alpha$ close to unity. They also devise an elasticity of substitution between housing and second-period consumption slightly larger than unity (about 1.3) corresponding to $\rho=0.24$. For simplicity, we follow Li \& Yao (2007) by confining ourselves to the case of unit elasticity setting $\rho=0$ which yields a CobbDouglas function for second-period utility. For this choice, the parameter $1-\beta$ can be interpreted as the share of housing expenditure in consumer income and Bajari, Chan, Krueger \& Miller $(2010)$ choose a value of $\beta \approx .77$. Given that housing is confined to the second period of life in our setup, we choose a smaller value $\beta=.67$. As in Hurd (1989), consumers' annual time discount is taken to be $1 / 1.011$ implying a discount factor $\gamma=0.70 \approx(1 / 1.011)^{35}$. We normalize incomes by setting $e^{o}=1$ and assume that first period incomes $\left\{e_{t}^{y}\right\}_{t \geq 0}$ follows a symmetric two-state Markov process with values in $\mathcal{E}^{y}=\left\{e_{\min }^{y}=1.425, e_{\max }^{y}=1.5\right\}$ and a time-invariant transition probability $\pi=0.2$. Thus the process is highly persistent with an $80 \%$ chance of retaining its current state and a $20 \%$ chance of switching to the opposite state. The fact that incomes are higher in the first than in the second period seems broadly consistent with empirical evidence, cf. Table 3 in Bajari, Chan, Krueger \& Miller (2010). ${ }^{9}$ Finally, our choice for $\kappa=1 / 3$ implies that housing costs make up slightly more than $10 \%$ of consumers' lifetime income. The initial values are set to $p_{0}=b_{0}=1$. Under this parametrization, the hypotheses of Lemma 4 hold for all $e^{y} \in \mathcal{E}^{y}$. Thus, for each fixed income stream $e_{t}^{y} \equiv e^{y} \in \mathcal{E}^{y}$, the dynamics (17a) converges to a unique steady state $\bar{q}\left(e^{y}\right)>0 .{ }^{10}$ In particular, the above parametrization implies that $R_{\min }=\mathcal{R}\left(\bar{q}\left(e_{\max }^{y}\right), e_{\max }^{y}\right)<1<\mathcal{R}\left(\bar{q}\left(e_{\min }^{y}\right), e_{\min }^{y}\right)=R_{\max }$ such that the necessary conditions for booms and busts of housing prices to occur are satisfied.

\section{Simulation results}

We simulate the model for $T=6000$ periods and display the time series in Figure 3 starting in $t=3000$ to capture the long run characteristics of the model. The left panel shows a time window of the housing price $p_{t}$ and the credit volume $b_{t}$. To relate movements in these variables to the 'fundamentals' of the economy we also depict the

\footnotetext{
${ }^{9}$ This is also consistent if we were to replace the pure exchange setting by a production economy where the young earn labor income and the old capital income. Empirical evidence then suggests that the former is about twice as large as the latter.

${ }^{10}$ We remark that the chosen parametrization guarantees positivity of steady states, which may fail to exist at all or $\bar{q}\left(e^{y}\right)<0$ for some $e^{y} \in \mathcal{E}^{y}$ under other parametrization.
} 
aggregate net income $e_{t}^{y}+e^{o}-\kappa$ which represents the total resources available in period $t$ net of housing costs. The right panel depicts the leverage ratio $b_{t} /\left(e_{t}^{y}+e^{o} R_{t}^{-1}\right)$ which measures the percentage share of loans backed by consumers' discounted lifetime incomes.

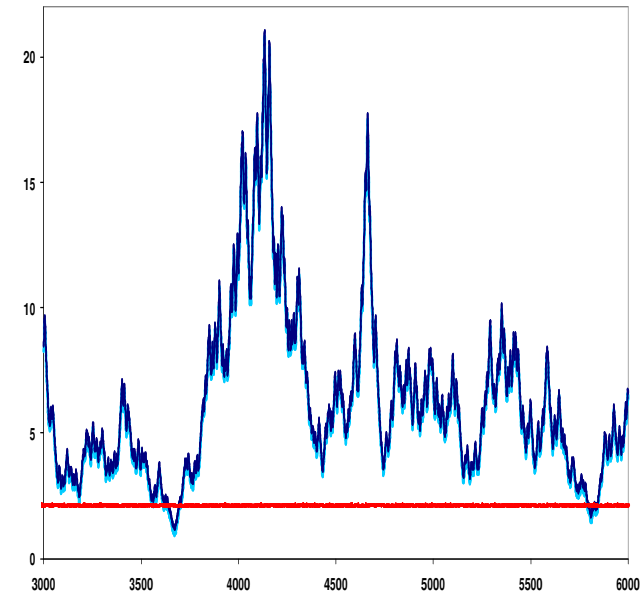

(a) $p_{t}, b_{t}$, and $e_{t}^{y}+e^{o}-\kappa$

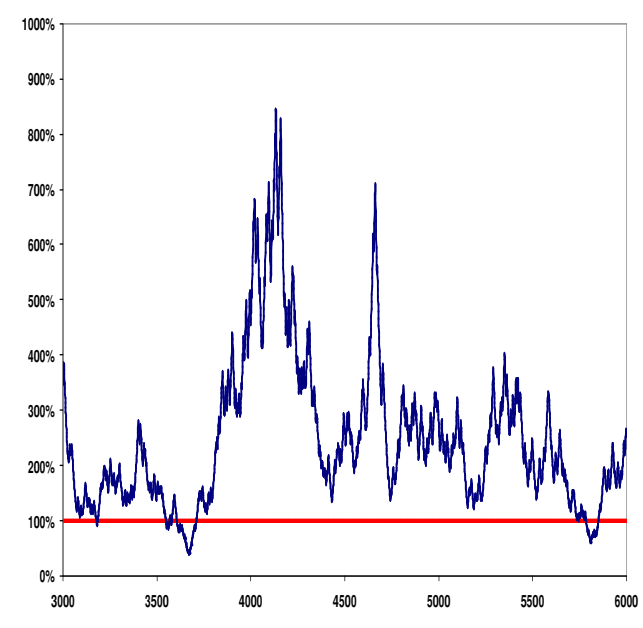

(b) $\frac{b_{t}}{e_{t}^{y}+e^{o} R_{t}^{-1}} \times 100 \%$

Figure 3: A boom-bust scenario

The figures confirm that the mechanism identified in the previous sections generates large and persistent movements in the housing price and the credit volume. These two variables are intimately interconnected and are almost impossible to distinguish in Figure 3(a). By our previous results, we know that $p_{t}-b_{t}$ follows a stationary stochastic process. We also see that for most time periods both the housing investment and the credit volume exceed the aggregate income by an order of magnitude.

To provide a quantitative illustration of these phenomena, consider the situation in period $t_{0}=4662$ where the bubble reaches a temporary peak. In this period, the young are in the low-income state receiving $e_{t_{0}}^{y}=1.425$ such that aggregate net income is $e_{t_{0}}^{y}+e^{o}-\kappa=2.09$. The credit volume supplied by banks is $b_{t_{0}}=17.23$ and the interest on loans is $2.7 \%$, i.e., $R_{t_{0}}=1.027$. The young buy houses at the price $p_{t_{0}}=17.24$ which is financed by taking a loan equal to $b_{t_{0}}$. This corresponds to a leverage ratio of $710 \%$. Moreover, the loan repayment $R_{t_{0}} b_{t_{0}}$ is about eighteen time as large as second-period non-housing income $e^{o}$. However, the next period's housing price $p_{t_{0}+1}=17.71$ allows consumers to repay their loan from the revenues of selling their houses at the end of period $t_{0}+1$. This confirms our earlier insight that a credit volume exceeding real incomes by an order of magnitude can still be sustained by a corresponding increase in housing values. In fact, the net flow from the young to old consumers, which is equal to the fundamental price of housing, is only $q_{t_{0}}=p_{t_{0}}-b_{t_{0}}=0.01$.

We remark that the same qualitative results were observed when first period income has an absolutely-continuous and state dependent probability distribution, which alter- 
nates between a high income distribution and a low income distribution with the same transition probability as before. Hence, the simplifying assumption of income following a two-state process is not essential for our numerical results.

\section{Conclusions}

In the absence of a banking sector the only intergenerational transfer of commodities in our model is from the young to old through the housing market. Consequently, housing values are bounded by young consumers' incomes. Introducing a banking sector adds an additional channel of intergenerational trade in the form of a credit market, which mediates a commodity transfer from the old to young. The combination of these channels permits each flow of intergenerational transfers to become arbitrarily large as long as the net flow remains bounded by consumers' incomes. This structure amplifies small but persistent income changes into large movements of housing prices and credit volumes, which are both non-stationary while a linear combination of them follows a stationary stochastic process. The presence of such a cointegration relationship is therefore an implication of the model that is testable empirically.

In our model, the boom in housing prices accompanied by expanding loan volumes occurs when the interest factor exceeds unity. Hence, the stationary endowment process implies that the interest rate is greater than the growth rate of the economy when bubbles emerge, a feature of the model shared by Arce \& López-Salido (2011), Caballero, Farhi, \& Hammour (2006), Martin \& Ventura (2012), and Ventura (2012). The boom comes to a halt when a higher income of the young causes the interest factor to drop below unity. This comovement between the interest rate and the credit volume occurs naturally in a model with inside money but may be at odds with the empirical observation that the cost of refinancing is relatively low in many bubble episodes. An interesting question that we leave for future research is whether this relationship reverses when the model includes outside money. Such an extension would also permit to investigate how monetary policies interact with the banking sector and the housing market.

Another feature of our exchange economy is that bubbles do not affect productive investment and hence consumer incomes. Whether bubbles in our framework remain welfare neutral in the presence of capital accumulation is also an interesting question to be explored. 


\section{A Mathematical Proofs}

\section{A.1 Proof of Lemma 1}

Let $e=\left(e^{y}, e^{o}\right) \in \mathcal{E}$ and $q<e^{y}$ be arbitrary but fixed. For brevity, set $\underline{q}:=\kappa-e^{o}$ and

$$
H\left(q_{1}\right):=v_{c}\left(q_{1}-\underline{q}, 1\right)\left(q_{1}-\kappa\right)+v_{h}\left(q_{1}-\underline{q}, 1\right), \quad q_{1}>\underline{q} .
$$

Since $v$ in (4) is homogeneous of degree $1-\alpha$, Euler's theorem for homogeneous functions implies $v_{c}(c, 1) c+v_{h}(c, 1)=(1-\alpha) v(c, 1)$ for all $c>0$ permitting us to write

$$
H\left(q_{1}\right)=(1-\alpha) v\left(q_{1}-\underline{q}, 1\right)-v_{c}\left(q_{1}-\underline{q}, 1\right) e^{o}, \quad q_{1}>\underline{q} .
$$

Since $\rho \geq 0$, the function $v$ satisfies the Inada condition $\lim _{c \rightarrow 0} v_{c}(c, 1)=\infty$. Thus,

$$
\lim _{q_{1} \rightarrow \underline{q}} H\left(q_{1}\right)=(1-\alpha) v(0,1)-e^{o} \lim _{q_{1} \rightarrow \underline{q}} v_{c}\left(q_{1}-\underline{q}, 1\right)=-\infty .
$$

Furthermore, the restrictions $\rho \geq 0$ and $\alpha<1$ together imply $\lim _{c \rightarrow \infty} c v_{c}(c, 1)=\infty$. Using this in (A.1) yields the right limit as

$$
\lim _{q_{1} \rightarrow \infty} H\left(q_{1}\right) \geq \lim _{q_{1} \rightarrow \infty} v_{c}\left(q_{1}-\underline{q}, 1\right)\left(q_{1}-\kappa\right)=\infty .
$$

Existence of the desired solution thus follows from (A.3), (A.4), and continuity of $H$. Uniqueness is a consequence of (A.2) and the concavity of $v$ which give the derivative

$$
H^{\prime}\left(q_{1}\right)=(1-\alpha) v_{c}\left(q_{1}-\underline{q}, 1\right)-v_{c c}\left(q_{1}-\underline{q}, 1\right) e^{o}>0 \text {. }
$$

\section{A.2 Proof of Lemma 2}

Since $F_{q_{1}}\left(q_{1}, q ; e\right)=-H^{\prime}\left(q_{1}\right)<0$ by (10) and (A.5) and $F$ is continuously differentiable, so is the implicit function $f$ by the Implicit Function Theorem. The partial derivative of (10) with respect to $q$ computes

$$
F_{q}\left(q_{1}, q ; e\right)=u^{\prime}\left(e^{y}-q\right)-q u^{\prime \prime}\left(e^{y}-q\right)=\left(e^{y}-q\right)^{-\alpha} \frac{e^{y}-(1-\alpha) q}{e^{y}-q}>0 .
$$

By the implicit function theorem $f^{\prime}(q)=-\frac{F_{q}\left(q_{1}, q ; e\right)}{F_{q_{1}}\left(q_{1}, q ; e\right)}>0$ where $q_{1}=f(q ; e)$. 


\section{A.3 Proof of Lemma 3}

By (15), the function $G$ can be written as $G(q)=D(q)-H(q)$ with $H$ being defined as in (A.2) and $D(q):=q u^{\prime}\left(e^{y}-q\right)=q\left(e^{y}-q\right)^{-\alpha}, \quad q<e^{y}$.

Consider first the behavior of the function $D$ whose derivatives satisfy

$$
\begin{aligned}
D^{\prime}(q) & =\frac{e^{y}-(1-\alpha) q}{\left(e^{y}-q\right)^{1+\alpha}}>(1-\alpha) \frac{e^{y}-q}{\left(e^{y}-q\right)^{1+\alpha}}=\frac{1-\alpha}{\left(e^{y}-q\right)^{\alpha}}>0 \\
D^{\prime \prime}(q) & =\frac{\alpha}{\left(e^{y}-q\right)^{2+\alpha}}\left(2 e^{y}-(1-\alpha) q\right)>\frac{\alpha(1-\alpha)}{\left(e^{y}-q\right)^{2+\alpha}}\left(e^{y}-q\right)>0 .
\end{aligned}
$$

The second inequality shows that $D$ is a strictly convex function while the first one implies that $D$ is strictly increasing with boundary behavior $\lim _{q \rightarrow e^{y}} D^{\prime}(q)=\infty$.

As shown in the proof of Lemma 1, the derivative of $H$ is given by (A.5) and, therefore, satisfies $H^{\prime}(q)>0$ and $\lim _{q \rightarrow \kappa-e^{o}} H^{\prime}(q) \geq(1-\alpha) \lim _{q \rightarrow \kappa-e^{o}} v_{c}\left(e^{o}-\kappa+q, 1\right)=\infty$. We claim that $H^{\prime}$ is a strictly decreasing function implying that $-H$ is strictly convex. The first term in (A.5) is strictly decreasing by strict concavity of $v$. It therefore suffices to show that $c \mapsto-v_{c c}(c, 1)$ is decreasing as well. Defining $g$ as in (2), direct calculations reveal that the second derivative of $v$ can be written as

$$
\begin{aligned}
-v_{c c}(c, 1) & =\frac{v_{c}(c, 1)}{c}\left[1-\rho-(1-\rho-\alpha) \frac{\beta c^{\rho}}{g(c, 1)^{\rho}}\right] \\
& =v_{c}(c, 1)\left[\frac{\alpha}{c^{1-\rho}} \frac{\beta}{g(c, 1)^{\rho}}+\frac{1-\rho}{c} \cdot \frac{(1-\beta)}{g(c, 1)^{\rho}}\right] .
\end{aligned}
$$

Recalling that $1-\rho \geq 0$, all three terms in (A.9) are positive and strictly decreasing functions of $c$ which implies that $c \mapsto-v_{c c}(c, 1)$ is decreasing as claimed.

Thus, $-H$ is a strictly convex function as claimed and $G$ being the sum of two (strictly) convex functions is also strictly convex. The boundary behavior of $G^{\prime}$ follows directly from the limits computed above and the monotonicity properties of $D$ and $-H$.

\section{A.4 Proof of Lemma 4}

(i) Using (15) in conjunction with (A.2), a routine calculation shows that $\lim _{q \rightarrow e^{y}} G(q)=$ $\lim _{q \rightarrow \kappa-e^{o}} G(q)=\infty$. Thus, $G\left(q_{\min }\right)<0$ implies that $G$ has a fixed point in each of the intervals $\left(\kappa-e^{o}, q_{\min }\right)$ and $\left(q_{\min }, e^{y}\right)$. By strict convexity and the boundary behavior of the first derivative (cf. Lemma 3 ), the map $G$ is strictly decreasing on $\left(\kappa-e^{o}, q_{\text {min }}\right)$ and strictly increasing on $\left(q_{\min }, e^{y}\right)$. Thus, there can be at most one fixed point in each of the two intervals.

(ii) It is obvious from (i) that $G^{\prime}(\bar{q})<0<G^{\prime}(\overline{\bar{q}})$. Utilizing the result from Lemma 2 and the definitions of $D$ and $H$ given in the proof of Lemma 4, this implies that $G^{\prime}(\bar{q})=D^{\prime}(\bar{q})-H^{\prime}(\bar{q})<0$ and $G^{\prime}(\overline{\bar{q}})=D^{\prime}(\overline{\bar{q}})-H^{\prime}(\overline{\bar{q}})>0$. Therefore,

$$
0<f^{\prime}(\bar{q})=\frac{D^{\prime}(\bar{q})}{H^{\prime}(\bar{q})}<1<\frac{D^{\prime}(\overline{\bar{q}})}{H^{\prime}(\overline{\bar{q}})}=f^{\prime}(\overline{\bar{q}})
$$


which implies the local stability properties asserted. The remaining inequalities follow from this and the uniqueness of the fixed points on the respective intervals.

\section{A.5 Proof of Lemma 5}

Assertion (i) follows immediately from Lemma 4(i). The result in (ii) is a consequence of local stability of $\bar{q}$ and Lemma 4(ii). Monotonicity of the sequence $\left\{q_{t}\right\}_{t>0}$ follows from this and Lemma 2.

\section{A.6 Proof of Theorem 1}

(i) Lemma 5 and $q_{0} \in \mathbb{Q}$ imply that $q_{t} \in \mathbb{Q}$ for all $t$ and $\lim _{t \rightarrow \infty} q_{t}=\bar{q}$. By (1) and (13), $b_{0}>0$ implies $b_{t}>0$ and, by (8) $p_{t}>0$ for all $t$ proving (i).

(ii) If $\mathcal{R}(\bar{q} ; e)>1$, then there exists $t_{0} \geq 0$ such that $\mathcal{R}\left(q_{t} ; e\right)>1$ for all $t \geq t_{0}$ by stability of $\bar{q}$. In fact, since $q \mapsto \mathcal{R}(q ; e)$ is strictly increasing (cf. Lemma 8) and $\left\{q_{t}\right\}_{t>0}$ converges monotonically, we have $\mathcal{R}\left(q_{t} ; e\right) \geq R_{t_{0}}:=\mathcal{R}\left(q_{t_{0}} ; e\right)>1$ for all $t \geq t_{0}$. Thus, $\lim _{t \rightarrow \infty} b_{t} \geq b_{t_{0}} \lim _{t \rightarrow \infty} R_{t_{0}}^{t-t_{0}}=\infty$ and $p_{t}=q_{t}+b_{t}>b_{t}$ for all $t$ gives $\lim _{t \rightarrow \infty} p_{t}=\infty$.

(iii) If $b_{0}=0$, then $b_{t}=0$ and $q_{t}=p_{t}$ for all $t$ and the claim follows from (i). If $b_{0}>0$ and $\mathcal{R}(\bar{q} ; e)<1$, the same arguments as in (ii) yield $\mathcal{R}\left(q_{t} ; e\right) \leq R_{t_{0}}:=\mathcal{R}\left(q_{t_{0}} ; e\right)<1$ for $t \geq t_{0}$. Thus, $0 \leq \lim _{t \rightarrow \infty} b_{t} \leq b_{t_{0}} \lim _{t \rightarrow \infty} R_{t_{0}}^{t-t_{0}}=0$ and $\lim _{t \rightarrow \infty} p_{t}=\lim _{t \rightarrow \infty} q_{t}=\bar{q}$.

\section{A.7 Proof of Lemma 6}

(i) The proof of Lemma 2 revealed that $F_{q_{1}}\left(q_{1}, q ; e\right)<0$ with $F$ defined in (10). Since $F_{e^{y}}\left(q_{1}, q ; e\right)=q u^{\prime \prime}\left(e^{y}-q\right)<0, q>0$, the claim follows from the Implicit Function Theorem.

(ii) Recall that fixed points are solutions to $G(q ; e)=F(q, q ; e)=0$. By (i), $G_{e^{y}}(q ; e)=$ $F_{e^{y}}(q, q ; e)<0$. As $\lim _{q \rightarrow \kappa-e^{o}} G(q ; e)=\lim _{q \rightarrow e^{y}} G(q ; e)=\infty$ implies $G_{q}(\bar{q} ; e)<0<$ $G_{q}(\overline{\bar{q}} ; e)$, the claim follows again from the Implicit Function Theorem.

\section{A.8 Proof of Lemma 7}

(i) We first show that $\overline{\mathbb{Q}}$ is self-supporting. Let $q \in \overline{\mathbb{Q}}$ be arbitrary. Then, using Lemma 4(ii) and the monotonicity properties of $f$ together with the definitions $(19 \mathrm{a}-\mathrm{c})$ we have for each $e^{y} \in \mathcal{E}$ :

$$
\left.\bar{q}_{\min }=f\left(\bar{q}_{\min } ; e_{\max }^{y}\right) \leq f\left(\bar{q}_{\min } ; e^{y}\right) \leq f\left(q ; e^{y}\right) \leq f\left(\bar{q}_{\max } ; e^{y}\right) \leq f\left(\bar{q}_{\max } ; e_{\min }^{y}\right)\right)=\bar{q}_{\max } .
$$

Thus, $f\left(q ; e^{y}\right) \in \overline{\mathbb{Q}}$. To prove that $\mathbb{Q}$ is self-supporting, let $q \in \mathbb{Q}$ and $e^{y} \in \mathcal{E}^{y}$ be arbitrary. The case $q \in \overline{\mathbb{Q}}$ is evident, so suppose first that $q \in\left(\bar{q}_{\max }, \overline{\bar{q}}_{\text {min }}\right)$. Then, 
by $(19 \mathrm{a}-\mathrm{c}), \bar{q}\left(e^{y}\right) \leq \bar{q}_{\max }<q<\overline{\bar{q}}_{\min } \leq \overline{\bar{q}}\left(e^{y}\right)$ which implies, by Lemma 4(ii) and monotonicity of $f$ that $\bar{q}\left(e^{y}\right)<f\left(q ; e^{y}\right)<q$. Thus, $f\left(q ; e^{y}\right) \in \mathbb{Q}$. Conversely, suppose $q \in\left(0, \bar{q}_{\text {min }}\right)$. Then, by $(19 \mathrm{a}-\mathrm{c}) 0<q<\bar{q}_{\text {min }} \leq \bar{q}\left(e^{y}\right)$ which implies $q<f\left(q ; e^{y}\right)<\bar{q}\left(e^{y}\right)$ by Lemma 4 (ii) and monotonicity of $f$. Thus, $f\left(q ; e^{y}\right) \in \mathbb{Q}$ again.

(ii) Let $q_{0} \in \mathbb{Q}$ be arbitrary. Define the sequences $\left\{\bar{q}_{t}\right\}_{t \geq 0}$ and $\left\{\underline{q}_{t}\right\}_{t \geq 0}$ by setting $\bar{q}_{0}=$ $\underline{q}_{0}=q_{0}$ and $\bar{q}_{t+1}:=f\left(\bar{q}_{t} ; e_{\min }^{y}\right)$ and $\underline{q}_{t+1}:=f\left(\underline{q}_{t} ; e_{\max }^{y}\right)$ for each $t \geq 0$. Then, by the monotonicity properties of $f, \underline{q}_{t} \leq q_{t} \leq \bar{q}_{t} \mathbb{P}$-a.s. for all $t \geq 0$ and the claim follows from $\lim _{t \rightarrow \infty} \underline{q}_{t}=\bar{q}\left(e_{\max }^{y}\right)=\bar{q}_{\min }$ and $\lim _{t \rightarrow \infty} \bar{q}_{t}=\bar{q}\left(e_{\min }^{y}\right)=\bar{q}_{\max }$.

\section{A.9 Proof of Theorem 2}

Lemma 7 ensures that $q_{t} \in \mathbb{Q} \subset\left(0, e_{\min }^{y}\right) \mathbb{P}$-a.s. for all $t \geq 0$. By $(1)$ and $(13), b_{0} \geq 0$ implies $b_{t} \geq 0$ by, (8), $p_{t} \geq q_{t}>0 \mathbb{P}$-a.s. for all $t \geq 0$.

\section{A.10 Proof of Lemma 8}

The claim follows directly by taking the partial derivatives of (13) and using Lemmata 2 and $6(\mathrm{i})$.

\section{A.11 Proof of Theorem 3}

(i) Suppose $R_{\min }>1$. Then, $\mathcal{R}(q ; e) \geq R_{\min }>1$ for all $q \in \overline{\mathbb{Q}}$ and $e \in \mathcal{E}^{y}$. Let $\hat{R}_{\text {min }}$ be a number between 1 and $R_{\text {min }}$. By continuity of $\mathcal{R}$, we can choose an open neighborhood $\hat{\mathbb{Q}} \subset \mathbb{Q}$ of $\overline{\mathbb{Q}}$ such that $\mathcal{R}(q ; e)>\hat{R}_{\text {min }}$ for all $q \in \hat{\mathbb{Q}}$ and $e \in \mathcal{E}^{y}$. Let $q_{0} \in \mathbb{Q}$ be arbitrary. By Lemma 7 (ii), there exists $t_{0}>0$ such that $q_{t} \in \hat{\mathbb{Q}}$ for all $t>t_{0} \mathbb{P}$-a.s. Hence, $R_{t}>\hat{R}_{\text {min }}>1$ for all $t>t_{0} \mathbb{P}$-a.s. and it follows from (1) that $\lim _{t \rightarrow \infty} b_{t} \geq \lim _{t \rightarrow \infty} b_{t_{0}}\left(\hat{R}_{\text {min }}\right)^{t-t_{0}}=\infty$. Since $q_{t}$ remains uniformly bounded, the limit of the process $\left\{p_{t}\right\}_{t \geq 0}$ follows from (8).

(ii) Similar to the previous part, choose a number $\hat{R}_{\max }$ between $R_{\max }$ and 1 and an open neighborhood $\hat{\mathbb{Q}} \subset \mathbb{Q}$ of $\overline{\mathbb{Q}}$ such that $\mathcal{R}(q ; e)<\hat{R}_{\max }<1$ for all $q \in \hat{\mathbb{Q}}$ and $e \in \mathcal{E}^{y}$. Let $q_{0} \in \mathbb{Q}$ be arbitrary. By Lemma $7\left(\right.$ ii), there exists $t_{0}>0$ such that $q_{t} \in \hat{\mathbb{Q}}$ for all $t>t_{0} \mathbb{P}$-a.s. Hence, $R_{t}<\hat{R}_{\max }<1$ for all $t>t_{0} \mathbb{P}$-a.s. and it follows that $0 \leq \lim _{t \rightarrow \infty} b_{t} \leq \lim _{t \rightarrow \infty} b_{t_{0}}\left(\hat{R}_{\max }\right)^{t-t_{0}}=0$. Finally, the previous result and (8) imply directly that $\lim _{t \rightarrow \infty}\left|p_{t}-q_{t}\right|=\lim _{t \rightarrow \infty}\left|b_{t}\right|=0$.

\section{References}

Arce, Ó. \& D. López-SAlido (2011): "Housing Bubbles", American Economic Journal: Macroeconomics, 3, 212-241. 
Bajari, P., P. Chan, D. Krueger \& D. Miller (2010): “A Dynamic Model of Housing Demand: Estimation and Policy Implications", NBER Working paper 15955.

Brock, W. A. \& L. J. Mirman (1972): "Optimal Growth and Uncertainty: The Discounted Case", Journal of Economic Theory, 4, 479-513.

Caballero, R. J., E. Farhi, \& M. L. Hammour (2006): "Stationary Markov Equilibria", American Economic Review, 96(4), 1159-1192.

Chen, H. \& C. Winter (2011): "Mortgage Loans, the Decline of Household Saving, and the Increase in Risk-Sharing", Discussion paper, University of Zurich.

Duffie, D., J. Geanakoplos, A. Mas-Colell \& A. Mclennan (1994): "Stationary Markov Equilibria", Econometrica, 62(4), 745-781.

Gale, D. (1973): "Pure Exchange Equilibrium of Dynamic Economic Models", Journal of Economic Theory, 6, 1236.

He, C., R. Wright, \& Y. Zhu (2011): "Housing and Liquidity", mimeo.

Hurd, M. (1989): "Mortality Risk and Bequests", Econometrica, 57, 173209.

Kocherlakota, N. R. (2011): "Two Models of Land Overvaluation and Their Implications", Working paper, Federal Reserve Bank of Minneapolis.

LI, W. \& R. YAO (2007): "The Life-Cycle Effects of House Price Changes", Journal of Money, Credit, and Banking, 39, 1375-1409.

Lustig, H. \& S. V. Nieuwerburgh (2005): "Housing Collateral, Consumption Insurance, and Risk Premia: An Empirical Perspective", Journal of Finance, 60(3), $1167-1219$.

Martin, A. \& J. Ventura (2012): "Economic Growth with Bubbles", American Economic Review, forthcoming.

Ortalo-Magné, F. \& S. Rady (2006): "Housing Market Dynamics: On the Contribution of Income Shocks and Credit Constraints", Review of Economic Studies, 73, 459-485.

Samuelson, P. (1958): "An Exact Consumption-Loan Model of Interest with or without the Social Contrivance of Money", Journal of Policital Economy, 66, 467482.

Ventura, J. (2012): "Bubbles and Capital Flows", Journal of Economic Theory, in press.

WANG, Y. (1993): "Stationary Equilibria in an Overlapping Generations Economy with Stochastic Production", Journal of Economic Theory, 61(2), 423-435. 


\section{Working Paper Series in Economics}

recent issues

No. 40 Marten Hillebrand and Tomoo Kikuchi: A mechanism for booms and busts in housing prices, May 2012

No. 39 Antje Schimke: Entrepreneurial aging and employment growth in the context of extreme growth events, May 2012

No. 38 Antje Schimke, Nina Teichert, Ingrid Ott: Impact of local knowledge endowment on employment growth in nanotechnology, February 2012

No. 37 Siegfried K. Berninghaus, Lora Todorova, Bodo Vogt: A simple questionnaire can change everything - are strategy choices in coordination games stable?, December 2011

No. 36 Matthias Duschl, Antje Schimke, Thomas Brenner, Dennis Luxen: Firm growth and the spatial impact of geolocated external factors - empirical evidence for German manufacturing firms, November 2011

No. 35 Mirja Meyborg: The impact of West-German universities on regional innovation activities - a social network analysis, October 2011

No. 34 Tom Broekel, Antje Schimke, Thomas Brenner: The effect of cooperative R\&D subsidies and subsidized cooperation on employment growth, October 2011

No. 33 Stephan Schosser and Bodo Vogt: The public loss game - an experimental study of public bads, August 2011

No. 32 Antje Schimke and Thomas Brenner: Temporal structure of firm growth and the impact of R\&D, July 2011

No. 31 Axel Schaffer: Appropriate policy measures to attract private capital in consideration of regional efficiency in using infrastructure and human capital, July 2011

No. 30 Stoyan V. Stoyanov, Svetlozar T. Rachev, Boryana Racheva-lotova, Frank J. Fabozzi: Fat-tailed models for risk estimation, May 2011 